\title{
Safety Study of Targeted and Localized Intra-Arterial Delivery of Gemcitabine in Patients with Locally Advanced Pancreatic Adenocarcinoma
}

\author{
Alexander S. Rosemurgy, ${ }^{1}$ Sharona B. Ross, ${ }^{1}$ Paul L. Vitulli, ${ }^{2}$ Reza Malek, ${ }^{3}$ Jaili Li, ${ }^{4}$ and Ramtin Agah ${ }^{5, *}$
}

\begin{abstract}
Purpose: This is a first-in-man safety study in locally advanced pancreatic cancer (LAPC) using a targeted intraarterial delivery catheter (RenovoCath ${ }^{\mathrm{TM}}$ ).

Methods: Twenty patients were enrolled in a four-stage dose escalation of intra-arterial, locally delivered gemcitabine, at doses up to $1000 \mathrm{mg} / \mathrm{m}^{2}$. Patients' symptoms and laboratory values were monitored for safety and tolerability. Secondary endpoints included the effect on tumor size, tumor markers, and survival.

Results: One hundred one treatments were administered to 20 patients. Five patients dropped out early due to adverse events or withdrawing consent. Serious adverse events and complications were as follows: sepsis $(n=3)$, grade 3 neutropenia $(n=3)$, guide-mediated vascular dissection $(n=3)$, and pulmonary toxicity $(n=1)$. There were no cases of elevated liver or pancreatic enzymes. All sepsis cases occurred in patients with biliary stent/ drains, prompting the addition of periprocedural treatment with antibiotics, which effectively prevented further sepsis in the study. Efficacy analysis was limited to 15 patients who received more than two treatments. Fiftyeight percent of these patients had a reduction in CA 19-9 tumor markers, 3 patients had tumor progression, 1 had partial response, and 11 showed disease stability. The survival rate at 12 months was $60 \%$.

Conclusions: The results demonstrate feasibility of localized and selective intra-arterial chemotherapy delivery to the pancreas utilizing the RenovoCath. With gemcitabine, this approach is safe, with the sole prerequisite of perioperative antibiotics for patients with prior biliary drainage/stent. Efficacy results suggest a survival benefit when compared to historical control, especially in patients with prior radiation therapy.
\end{abstract}

Keywords: gemcitabine; intra-arterial delivery; locally advanced pancreatic cancer; catheter

\section{Introduction}

Improving the survival curve of pancreatic cancer has been a clinical challenge, with an average median survival time stalled at 9-12 months from time of diagnosis. ${ }^{1}$ Most patients are diagnosed in the later stages ( 3 and 4 ) when they are ineligible for surgical resection, currently the only potential for a cure. The last 3 years have seen new approaches, including the use of combination chemotherapy (fluorouracil [5-FU], leucovorin, irinotecan, and oxaliplatin or gemcitabine plus Abraxane) for the treatment of stage 4 (metastatic) disease, and modest gains in survival with an increase in median survival of $8-16$ weeks. ${ }^{2,3}$

However, there has been little progress in treating patients with locally advanced disease (LAPC [locally advanced pancreatic cancer]). The only class one indicated treatment for these patients remains systemic gemcitabine, which was approved 18 years ago. Various other treatment strategies have been used, including the previously mentioned combination chemotherapy approved for patients with stage 4 disease, a chemoradiation regimen and, more recently, registry data that 
support the use of irreversible electroporation both with and without resection. ${ }^{4-11}$

One major cause of treatment failure for pancreatic cancer appears to be the inability of systemic chemotherapy to penetrate the tumor tissue. Koay et al. demonstrated that patients with poor prognosis may have two rate-limiting steps affecting drug efficacy ${ }^{12}$ : (1) poor penetration of the drug through the extracellular matrix (poor perfusion of dense matrix) and (2) poor intracellular metabolism of the drug, in the case of gemcitabine.

In line with these thoughts, various methods are under investigation to overcome the poor perfusion into these tumors and improve drug penetration and subsequent clinical outcomes. These include use of agents/drugs to increase the permeability of the extracellular matrix, binding of the drug to carriers to enhance uptake, and directly accessing the tumor through laparoscopy/laparotomy. ${ }^{13-15}$

Another recent approach involves the use of a novel catheter, the RC-120 (RenovoRx, Los Altos, $\mathrm{CA}$ ), to isolate segments of the major blood vessels around the tumor and, using localized perfusion to deliver high concentrations of the drug directly into the tumor. The advantages of localized intra-arterial (IA) drug therapy to the pancreas, shown in animal study, ${ }^{16}$ are several, including the following:

(1) A much higher concentration of the drug reaches the tumor when delivered locally, rather than systemically.

(2) The mechanism of drug delivery is independent of visible feeders to the tumor.

(3) Systemic exposure to the drug, which is a ratelimiting step in drug escalation dosing, is reduced.

The RC-120 is a dual-balloon catheter introduced through the femoral artery into the arterial segment of interest. The dual-balloon mechanism isolates blood flow in the region and allows the infusion of drug into the isolated region through an infusion port located between the two balloons. In this study, we show the results of the first-in-man study, which evaluated the safety of using the RC-120 catheter in patients with LAPC. The study used a dose escalation regimen of gemcitabine starting at $250 \mathrm{mg} / \mathrm{m}^{2}$ and increasing up to $1000 \mathrm{mg} /$ $\mathrm{m}^{2}$ (the standard dose for intravenous infusion), as tolerated. The primary endpoints were to establish the maximum tolerated dose of gemcitabine administered intra-arterially to the pancreatic tumor and to test the safety and tolerability of gemcitabine administered intra-arterially to pancreatic tumors using the RC-120 device. The secondary endpoints of the study included overall response (as assessed using Response Evaluation Criteria in Solid Tumors [RESIST] criteria), tumor marker reduction, and overall survival.

\section{Methods}

This study was carried out at two institutions, El Camino Hospital in Mountain View, California, and Florida Hospital in Tampa Bay, and approved by the local IRB at both institutions. Eligible patients had a diagnosis of stage 3 locally advanced unresectable pancreatic cancer (based on NCCN guidelines). Patients with prior treatments were eligible, with the exception of prior pancreatic resection surgery. They must have had a Karnofsky score of 70 or greater at the time of enrollment and been able to undergo catheterization procedure. Twenty patients at two centers were enrolled in a four-stage dose escalation study of gemcitabine up to $1000 \mathrm{mg} / \mathrm{m}^{2}$. Each patient underwent up to eight IA treatments. Standard laboratory values, including liver and pancreatic enzymes, blood counts, and clinical symptoms were monitored to assess doselimiting toxicities (DLTs). Feasibility and safety of repeated IA treatments were also assessed. Secondary endpoints that were assessed included the effect on tumor markers and on tumor size as measured by imaging.

Treatment was delivered in a 28 -day cycle and consisted of two IA doses of gemcitabine, with the first dose on Day 1 and the second dose on Day 15 of the cycle. There was an additional week of rest between each cycle. Intrapatient dose escalation was followed, and the first dose escalation occurred at the beginning of Cycle 2 (on Day 36) at a dose of $500 \mathrm{mg} / \mathrm{m}^{2}$ if no DLTs were experienced in Cycle 1 (Fig. 1). Dose escalation occurred at the beginning of Cycle 3 to a dose of $750 \mathrm{mg} / \mathrm{m}^{2}$ (on Day 71) if no DLTs were experienced in Cycle 2. The dose was further escalated to $1000 \mathrm{mg} / \mathrm{m}^{2}$ (on Day 106) at the beginning of Cycle 4 if no DLTs were experienced in Cycle 3.

Each patient underwent CT angiography (CTA) of the abdomen before the first procedure. The target vessel was selected based on best proximity to the tumor before the interventional procedure. The arteries targeted included the celiac artery, the splenic artery, the common hepatic artery, the gastroduodenal artery (GDA), and the superior mesenteric artery (SMA), based on tumor location. As some tumors abutted more than one vessel, an alternative treatment site was chosen in sequential treatments. Angiography 


\begin{tabular}{|c|c|c|c|}
\hline Cycle & Day & Chemotherapy & Dose \\
\hline $1^{*}$ & 1 & Gemcitabine & $250 \mathrm{mg} / \mathrm{m}^{2}$ \\
& 8 & & \\
& 15 & Gemcitabine & $250 \mathrm{mg} / \mathrm{m}^{2}$ \\
& 22 & & \\
\hline 2 & 1 & Gemcitabine & $500 \mathrm{mg} / \mathrm{m}^{2}$ \\
& 8 & Gemcitabine & $500 \mathrm{mg} / \mathrm{m}^{2}$ \\
& 15 & & \\
\hline 3 & 22 & Gemcitabine & $750 \mathrm{mg} / \mathrm{m}^{2}$ \\
& 1 & & $750 \mathrm{mg} / \mathrm{m}^{2}$ \\
& 15 & Gemcitabine & \\
\hline 4 & 22 & & $1000 \mathrm{mg} / \mathrm{m}^{2}$ \\
& 1 & Gemcitabine & \\
& 29 & Gemcitabine & $1000 \mathrm{mg} / \mathrm{m}^{2}$ \\
& 22 & & \\
\hline & 29 & & \\
\hline
\end{tabular}

FIG. 1. Treatment study regimen for IA treatment. Cycle 1 started at the lowest dose of gemcitabine ( $250 \mathrm{mg} /$ $\mathrm{m}^{2}$ ). Subsequent cycles were dose escalated by $250 \mathrm{mg} / \mathrm{m}^{2}$ each cycle if a DLT was not experienced during that cycle, up to a maximum dose of $1000 \mathrm{mg} / \mathrm{m}^{2}$. ${ }^{*}$ Starting with the ninth enrolled patient, the dose for the first cycle increased to $500 \mathrm{mg} / \mathrm{m}^{2}$. DLT, dose-limiting toxicity; IA, intra-arterial.

was performed using standard techniques, followed by the introduction of a guiding sheath to the target vessel. Patients were heparinized intravenously at a dose of $50 \mathrm{mg} / \mathrm{kg}$ during the procedure, and subsequently, the RC-120 device was introduced to the target vessel using prior CTA images to determine the ideal landing zone in the target vessel. This was done through $3 \mathrm{D}$ reconstruction of the tumor boundaries (TeraRecon Software) and then overlaying the pictures on CTA images. The arterial landmarks on the CTA were then used to identify the balloon landing zone before the procedure. During the procedure, once the catheter was in position and the dual balloons inflated, gemcitabine was infused over $20 \mathrm{~min}$ at the rate of $6 \mathrm{~mL} / \mathrm{min}$ (total of 120 $\mathrm{mLs})$. After the infusion was completed, the catheter and guiding sheath were removed, and standard closure technique to the access site was utilized. Patients stayed overnight for observation and requisite blood draws. Once IA therapy was completed, the patients were subsequently managed at the discretion of their physician.

\section{Statistical technique}

Kaplan-Meier method was used to calculate survival over time. Log-Rank test was used to compare survival estimates across groups. Fisher's exact test was used to make binary comparisons looking at age, sex, number of treatments, tumor size, and prior treatment and treatment outcome.

\section{Results}

Twenty patients with LAPC received dose-escalated gemcitabine delivered intra-arterially through the RC120 device at two centers over a 14-month period starting in May 2015. Baseline demographic and clinical characteristics are listed in Table 1 . The median age of patients was 70 years (range: $51-84$ years), including 9 men and 11 women. Nine patients were treatment naive, six had received prior chemoradiation, and five had prior chemotherapy. Eighty-five percent of patients presented with pancreatic head/uncinate process lesions and $15 \%$ presented with body/tail lesions. Nine of the 20 patients had a biliary stent or drain in place before the first IA procedure. Median follow-up for all patients was 12 months from the time of study initiation. During the course of the study, a total of 101 cycles of IA chemotherapy were administered to 20 patients.

The two most common serious complications associated with the procedure and resulting in early dropout were sepsis and vascular dissection (Table 2). We witnessed three episodes of sepsis during the study, 
Table 1. Patient Characteristics

\begin{tabular}{lcr}
\hline Characteristic & No. & $\%$ \\
\hline Age, years & 70 & \\
$\quad$ Median & $51-84$ & \\
Range & & 45 \\
Sex & 9 & 55 \\
$\quad$ Male & 11 & \\
Female & & 0 \\
Stage of tumor & 0 & 0 \\
I & 0 & 100 \\
II & 20 & 0 \\
III & 0 & 55 \\
IV & & 45 \\
Prior treatment & 11 & 25 \\
Yes & 9 & 75 \\
No & & \\
Prior chemotherapy only & 5 & 30 \\
Yes & 15 & 70 \\
No & & 0 \\
Prior chemoradiation & 6 & 100 \\
$\quad$ Yes & 14 & \\
No & & \\
Prior surgery & 0 & \\
Yes & 20 & \\
No & & \\
\hline
\end{tabular}

A total of 20 patients were enrolled between May 2015 and July 2016 at two participating sites. The median age was 70 , with 9 men and 11 women. At the time of enrollment, 11 patients had received prior treatment before joining the study.

two of which resulted in early patient dropout. Two of the three cases occurred after the very first treatment. The incidences of sepsis were limited to patients who had previous biliary obstructions that required prior interventions for drainage of the biliary tract (i.e., stents and or drains) and treatment in the hepatic artery. There was no incidence of sepsis in patients who did not have prior external manipulation of the biliary duct. This suggests colonization of the tract as a potential substrate for cholangitis and sepsis follow-

Table 2. Serious Adverse Events

\begin{tabular}{ll}
\hline Parameter & No. of patients \\
\hline Vascular & \\
$\quad$ Visceral arterial dissection & \\
$\quad$ Requiring termination of further treatment & 2 \\
$\quad$ Continuing further treatment & 1 \\
$\quad$ Vascular access complication & \\
$\quad$ Hematoma (self-limited, conservative & 1 \\
$\quad$ management) & \\
Nonvascular & \\
Pulmonary distress & 1 \\
Sepsis & 3 \\
Gastritis & 1 \\
\hline
\end{tabular}

Sepsis and vascular dissection were the most common complications associated with the procedure that contributed to early dropout. ing arterial chemotherapy. Of note, in prior reports of patients with transarterial delivery of chemotherapy to the liver, a similar mechanism of sepsis has been described. ${ }^{17}$ Instituting a preoperative and one-week postoperative prophylactic regimen of antibiotics toward the latter half of our study prevented any further incidence of sepsis in our cohort.

All cases of arterial dissection involved manipulation and placement of the guiding sheath into the tortuous area of vessel or segments involving tumor impingement on the vessel wall. We witnessed three such dissections, two of which resulted in early dropout from the study; the third was treated with a stent. None of dissections resulted in clinical sequelae, likely due to the redundant nature of the arterial vasculature.

Other rare complications $(n=1)$ are summarized in Table 2. Notable among these complications is one case of pulmonary toxicity following the sixth treatment, a rare, but known, adverse event associated with gemcitabine. ${ }^{18}$ The reaction was self-limiting, requiring a onenight hospital stay with subsequent discharge of the patient after improvement of his pulmonary status; it did, however, obviate any further treatment in the study.

It is noteworthy that, while a primary focus of the safety study was to assess the potential for pancreatitis and/or hepatitis as a possible result of local occlusion of blood flow to the treatment area and delivery of highdose chemotherapy, we observed no incidence of either.

In terms of hematological side effects (Table 3 ), three patients experienced Grade 3 neutropenia 1 week after drug infusion (15\%), none of whom required any intervention and all of whom returned to baseline values before the next treatment. We had three cases of grade 1

Table 3. Toxicities (Grade 3 and Grade 4)

\begin{tabular}{|c|c|c|c|c|}
\hline \multirow[b]{2}{*}{ Toxicity } & \multicolumn{2}{|c|}{ Grade 3} & \multicolumn{2}{|c|}{ Grade 4} \\
\hline & $\begin{array}{l}\text { No. of } \\
\text { patients }\end{array}$ & $\%$ & $\begin{array}{c}\text { No. of } \\
\text { patients }\end{array}$ & $\%$ \\
\hline \multicolumn{5}{|l|}{ Hematologic } \\
\hline Neutropenia & 3 & 15 & 0 & 0 \\
\hline Thrombocytopenia & 0 & 0 & 0 & 0 \\
\hline Leukopenia & 0 & 0 & 0 & 0 \\
\hline \multicolumn{5}{|l|}{ Nonhematologic } \\
\hline Bilirubin & 0 & 0 & 0 & 0 \\
\hline Hyperglycemia & 3 & 15 & 0 & 0 \\
\hline Elevated liver function test & 0 & 0 & 0 & 0 \\
\hline Elevated pancreatic enzymes & 0 & 0 & 0 & 0 \\
\hline Gastritis & 1 & 5 & 0 & 0 \\
\hline
\end{tabular}

Three patients (15\%) experienced grade 3 neutropenia 1 week after drug infusion. These cases involved drug infusion in arteries with at least one visible side branch. 
Table 4. Efficacy: Tumor Response (Clinical and or Radiological Progression), RECIST Criteria CT Response, CA19-9

\begin{tabular}{lcr}
\hline Parameter & No. of patients & $\%$ \\
\hline Stable disease & 11 & 73 \\
Progression & 3 & 20 \\
Partial response & 1 & 7 \\
CA 19-9 reduction & 9 & 60 \\
$>20 \%$ CA 19-9 reduction & 5 & 33 \\
\hline
\end{tabular}

Of the 15 patients who received more than two treatments, 11 had stable disease, 3 experienced tumor progression, and 1 had partial response. Nine patients displayed a reduction in CA 19-9 tumor markers, in five of which it was greater than $20 \%$.

RESIST, Response Evaluation Criteria in Solid Tumors.

transient hyperglycemia. All six cases of nausea were grade 1.

Overall, five patients dropped out early (two treatments or less) from the study. The causes of dropout were sepsis, arterial dissection, withdrawing consent, or a combination thereof. The other 15 patients received on average 5.6 treatments, with seven patients completing all eight treatments.
Efficacy

We limited our efficacy analysis to the 15 patients who had received more than one cycle of two treatments. Reasons for early dropout are as discussed above. In this cohort, five patients had no prior treatment, five had prior chemotherapy, and five had chemoradiation before their IA chemotherapy treatment.

Based on RECIST criteria, 3 patients experienced tumor progression, 1 had partial responses, and 11 demonstrated disease stability at the end of the study 5 months post-treatment initiation (Table 4). Twelve patients had measurable CA 19-9 levels. Overall, 58\% had some reduction of their CA19-9 tumor markers and $35 \%$ had a greater than $20 \%$ reduction in CA $19-9$.

The survival rate was $60 \%$ at 12 months and $43 \%$ at 24 months from the time of diagnosis in these patients. Notably, in the subgroup of seven patients that completed all eight treatments, we observed a survival rate of $78 \%$ at 12 months and $53 \%$ at 24 months.

Last, it appears that prior radiation positively impacts the efficacy of our treatment. Five out of the 15 patients analyzed received radiation treatment before

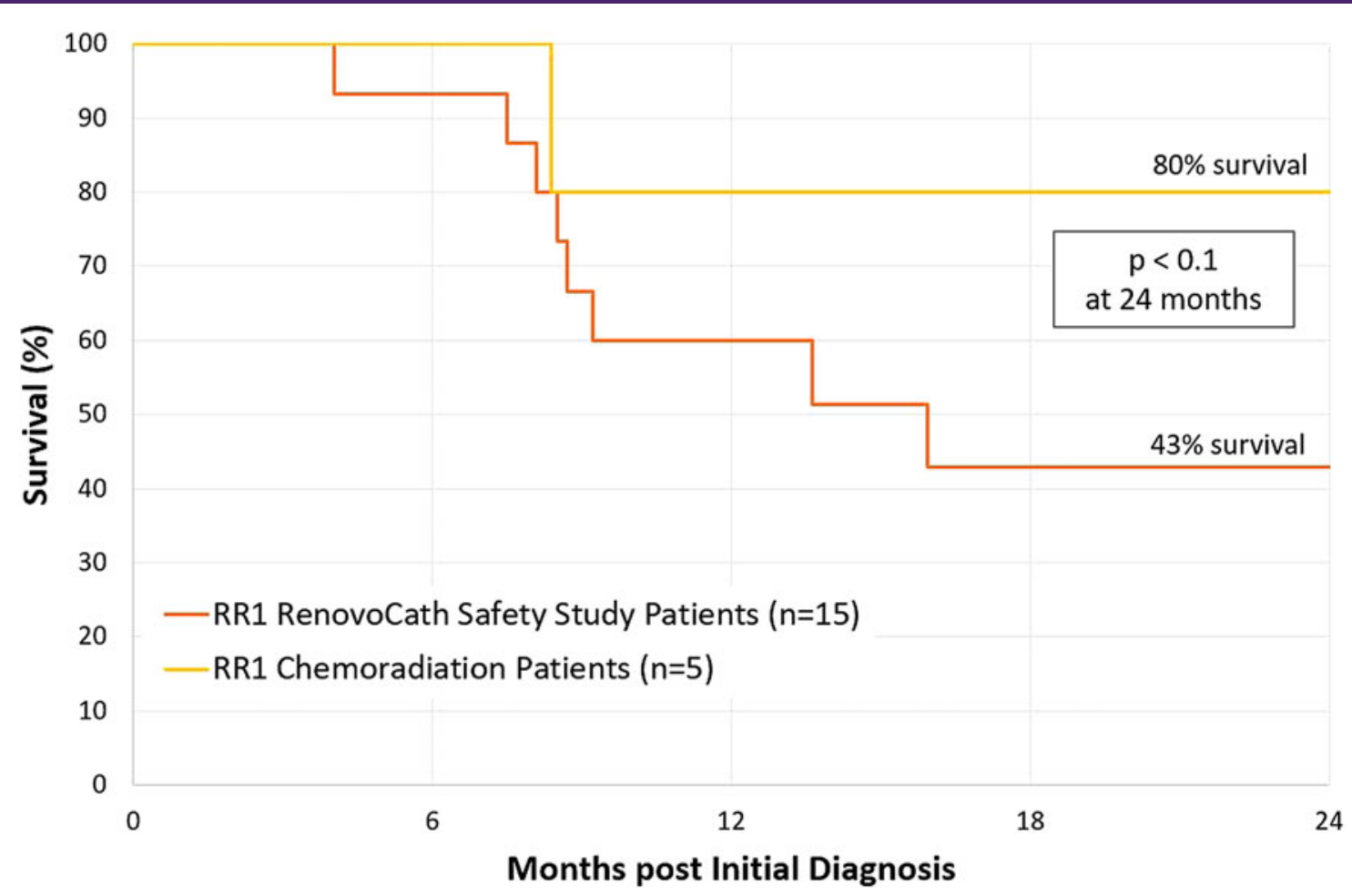

FIG. 2. Survival curve. The median survival from the time of diagnosis is $43 \%$ for all 15 patients at 24 months. From these 15 patients, the subset of patients with prior chemoradiation treatment $(n=5)$ had a median survival of $80 \%$ at 24 months. 


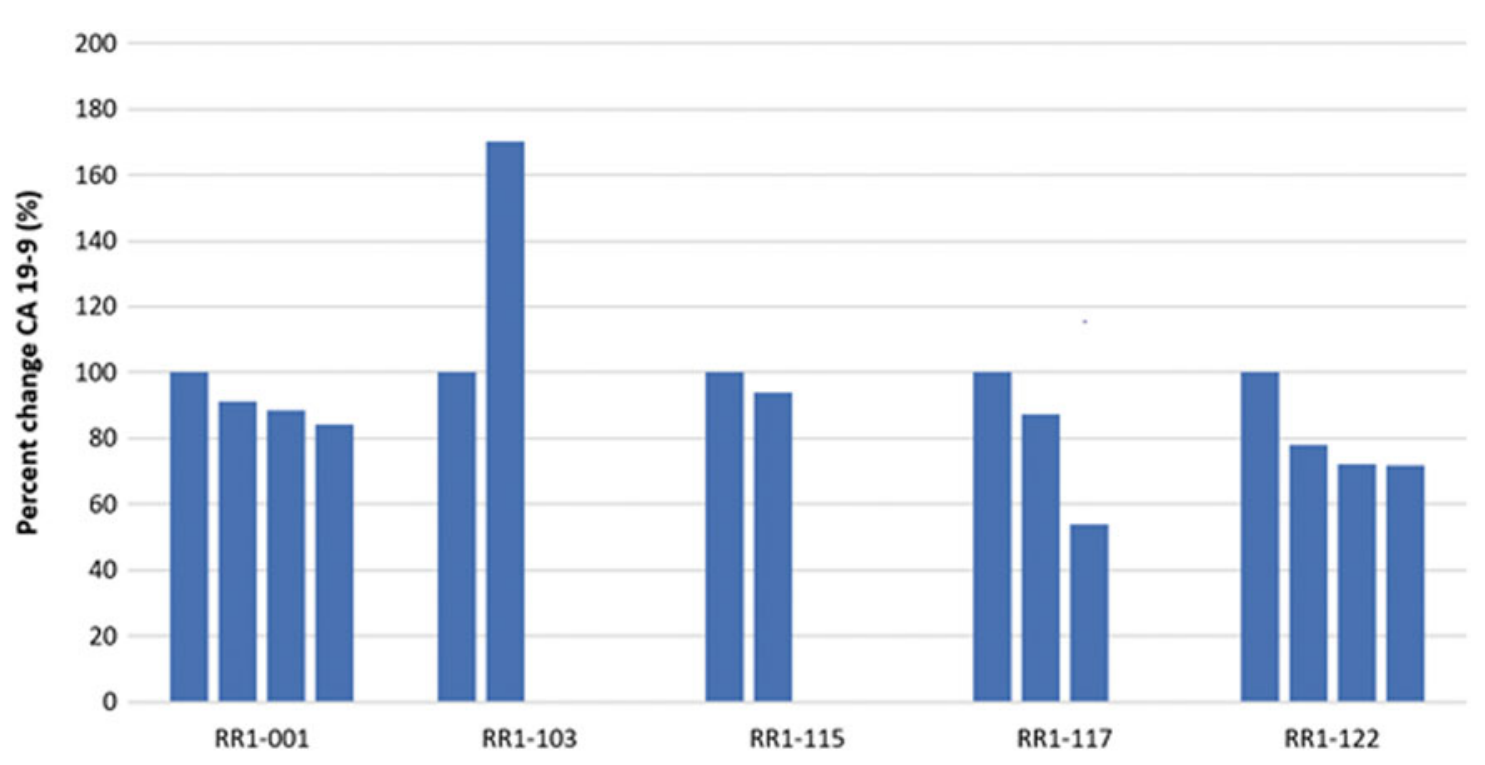

FIG. 3. Change in A 19-9 following RenovoCath IA treatments in patients with prior chemoradiation. Four of the five patients with prior chemoradiation had a consecutive drop in CA 19-9 tumor markers. Each bar represents one treatment cycle.

treatment with the RC-120. Of these five, four survived beyond 2 years from the time of diagnosis with survival of $80 \%$ at 24 months (Fig. 2). Furthermore, those who received prior chemoradiation treatment had the best response in terms of consecutive reduction of tumor marker after each cycle treatment (shown in Fig. 3$80 \%$ of patients in this cohort versus $10 \%$ in all other patients, $p<0.05$ ).

\section{Discussion}

Commonly, a goal for patients with LAPC is to determine if upfront chemotherapy or combination chemoradiation can reduce the stage of the tumor and allow for resection.

However, the majority of patients with LAPC are ineligible for resection and ultimately receive systemic therapy and local palliative therapies alone. As such, nonsurgical approaches remain the mainstay of therapy in these patients. ${ }^{1}$

A key limiting factor for clinical efficacy of therapy in LAPC patients is inefficient drug delivery to the cancer cells, due to the hypovascular nature of pancreatic cancer tumors as well as the dense matrix deposited around the tumor cells. ${ }^{12,19,20}$ As such, the current treatment paradigm for patients with LAPC involves treatment regimens that go beyond systemic drug therapy and, instead, uses modalities that address the disease locally and directly, including radiation and treatment based on electroporation. Radiation, including stereotactic body radiation therapy, despite showing early promising results in stabilizing the primary tumor, has not demonstrated significant survival benefit in recent trials. ${ }^{6,21}$ Last, the new modality based on electroporation for local disease control has shown promising registry results, but prospective data are lacking, and the application is mostly limited to patients already being considered for surgery and resection (i.e., borderline resectable patients). ${ }^{11}$

Previous studies using nonselective transarterial chemotherapy (TAC) with drug therapy for patients with pancreatic cancer have shown modest positive results $^{22,23}$; a meta-analysis of 298 patients (both stage 3 and 4) treated with TAC using gemcitabine showed a survival benefit of $30 \%$ at 1 year, compared with systemic gemcitabine. ${ }^{24}$ This modest benefit achieved with IA infusion can potentially be improved if more selective drug delivery to the pancreas itself is achieved, rather than nonselective arterial infusion leading to drug flowing into other organs such as the liver and the spleen. However, selective engagement of tumor feeders in LAPC is not feasible due to the inability to identify feeders by angiography. The approach with the current technology can potentially overcome both the above limitations (1) by blocking the flow of drugs proximal and distal to 
the pancreatic tissue and (2) by using microperfusion/ microdiffusion through the vessel wall to deliver the drugs into the tissue, obviating the need for identifying and cannulating tumor feeders.

Here, as part of a safety study, we present the use of gemcitabine in the first-in-human application of this technology. In this dose escalation study, we demonstrate that local delivery of gemcitabine using the RC120 device is safe up to a dose of $1000 \mathrm{mg} / \mathrm{m}^{2}$. The major serious adverse events associated with this approach were sepsis (seen in patients with prior biliary stent/drain) and guiding sheath-mediated dissection. The incidence of former is similar to the occurrence of sepsis in transarterial chemoembolization (TACE) treatments for liver tumors; in our subsequent treatment of patients with prior biliary drains/stents, the use of perioperative antibiotics (the approach taken to prevent sepsis in TACE treatments for liver tumors) prevented further incidences of sepsis in these patients (including unpublished data of 17 subsequent patients treated since the completion of safety study).

Regarding guiding sheath-mediated dissection, as this is the first-in-man use of this catheter, the expected learning curve for use of the technique/technology is not inconsistent with this reported complication. ${ }^{25}$ We expect that the number of dissections will decrease as procedural experience with the technique increases.

There were 6 relatively low incidences of grade 3 neutropenia in 101 IA treatment ( 2 incidence in 3 patients each). There was no correlation between the dose of gemcitabine and incidence of neutropenia in this small subset. Of note, all cases of neutropenia involved drug infusion in an area of the artery where there was at least one visible side branch, presumably resulting in systemic washout of locally delivered drugs. With local therapy, the incidence if neutropenia appears favorable compared to historical data for systemic gemcitabine $(15 \%$ vs. $27 \%){ }^{3}$

There was one incidence of pulmonary toxicity/ pneumonitis. This patient had a unique anatomy with chronic occlusion of the celiac axis due to atherosclerotic disease and retrograde flow of the hepatic arteries through the GDA, through the SMA. As the tumor was located in the pancreatic head, we could not achieve direct isolation of the vessel segment abutting the tumor; as such, the decision was made to deliver the drug across the pancreatoduodenal connection by isolating the SMA with expected retrograde flow of drug into the GDA. We assume that the free flow of the drug across the GDA may have introduced gemcitabine into the pulmonary venous system through an arteriovenous malformation.

In our cohort, based on CT and clinical findings, we were able to demonstrate disease stability in $80 \%$ of the patients, on average 5.0 months after first treatment (3 out of 15 patients had progressive disease before 5.0 months), and $35 \%$ of the patients showed greater than a $20 \%$ reduction in CA $19-9$.

The overall survival for this cohort appears encouraging when compared with systemic gemcitabine using historical controls (Fig. 2). Despite multiple treatment pathways being explored for patients with LAPC, there are no randomized trials for some of the more contemporary multidrug regimens that have been shown to be superior to single-agent gemcitabine. Nevertheless, registry data (excluding borderline resectable patients) yield median survival of 13-16 months, which compares favorably to our results. ${ }^{6,26}$ However, the efficacy portion of this study is limited by the small sample size and, furthermore, unintended bias toward a positive result in an industry-sponsored study.

Of note, patients with prior chemoradiation seemed to receive the greatest clinical benefit from IA therapy with RC-120 catheter, as measured both by median survival (Fig. $2 p<0.1$ ), and consecutive CA 19-9 reduction after each treatment (Fig. 3). This finding forms the basis of a hypothesis that prior chemoradiation enhances the effects of IA treatment that requires further investigation in both pre-clinical and clinical studies.

In summary, the results of this First-in-Man study demonstrate the feasibility of localized and selective IA chemo delivery to the pancreas utilizing the RC-120 Catheter. With the use of gemcitabine, this approach is safe, with the sole prerequisite of perioperative antibiotic treatment for patients with prior biliary drainage/stent. The preliminary clinical efficacy is encouraging and should be investigated in subsequent phase $2 / 3$ studies.

\section{Acknowledgments}

The authors listed were all the principal and coprincipal investigators at the El Camino Hospital in Mountain View, California, and Florida Hospital in Tampa Bay, and had significant input in the conduct of the work and preparation of the article. The study was funded by RenovoRx (Los Altos, California).

\section{Author Disclosure Statement}

The study was funded by RenovoRx (Los Altos, CA), the manufacturer of the catheter. Two of the six 
authors (R.A. and R.M.) own stock in RenovoRx and another author (P.V.) received a travel grant from the company during the conduct of the study. The article was entirely written by the authors, with no input from RenovoRx.

\section{References}

1. Ryan D, Hong T, Bardeesy N. Pancreatic adenocarcinoma. N Engl J Med. 2014;371:1039-1049.

2. Conroy T, Desseigne F, Ychou M, et al. FOLFIRINOX versus Gemcitabine for Metastatic Pancreatic Cancer for the Groupe Tumeurs Digestives of Unicancer and the PRODIGE Intergroup*. Reims (OB Nantes (JB Lyon (DP-V). Cent N Engl J Med. 2011;364:1817-1825.

3. Von Hoff DD, Ervin T, Arena FP, et al. Increased survival in pancreatic cancer with nab-paclitaxel plus gemcitabine. N Engl J Med. 2013;369:1691-1703.

4. Philip PA, Lacy J, Dowden SD, et al. LAPACT: an open-label, multicenter phase II trial of nab-paclitaxel (nab-P) plus gemcitabine (Gem) in patients (pts) with locally advanced pancreatic cancer (LAPC). Presentations by Philip Agop Philip. J Clin Oncol. 2016;suppl 4S:TSP477.

5. Hosein PJ, Macintyre J, Kawamura $C$, et al. A retrospective study of neoadjuvant FOLFIRINOX in unresectable or borderline-resectable locally advanced pancreatic adenocarcinoma. BMC Cancer. 2012;12:199.

6. Hammel P, Huguet F, Van Laethem J-L, et al. Effect of chemoradiotherapy vs chemotherapy on survival in patients with locally advanced pancreatic cancer controlled after 4 months of gemcitabine with or without erlotinib: the LAP07 randomized clinical trial. doi: 10.1001/jama .2016 .4324

7. Small Jr. W, Berlin J, Freedman GM, et al. Full-dose gemcitabine with concurrent radiation therapy in patients with nonmetastatic pancreatic cancer: a multicenter phase II trial. J Clin Oncol. 2008;26:942-947.

8. Sr PJL, Feng Y, Cardenes $\mathrm{H}$, et al. Gemcitabine alone versus gemcitabine plus radiotherapy in patients with locally advanced pancreatic cancer: an Eastern Cooperative Oncology Group Trial. J Clin Oncol 2011;29. doi: 10.1200/JCO.2011.34.8904.

9. Faris JE, Blaszkowsky LS, Mcdermott S, et al. FOLFIRINOX in Locally Advanced Pancreatic Cancer: the Massachusetts General Hospital Cancer Center experience. Oncologist 2013;18:543-548.

10. Chauffert B, Mornex F, Bonnetian F, et al. Phase III trial comparing intensive induction chemoradiotherapy (60 Gy, infusional 5-FU and intermittent cisplatin) followed by maintenance gemcitabine with gemcitabine alone for locally advanced unresectable pancreatic cancer. Definitive results of the 2. Ann Oncol. 2008;19:1592-1599.

11. Martin RCG. Treatment of 200 locally advanced (stage III) pancreatic adenocarcinoma patients with irreversible electroporation safety and efficac. Ann Surg. 2016;262:486-494.

12. Koay EJ, Truty MJ, Cristini V, et al. Transport properties of pancreatic cancer describe gemcitabine delivery and response. J Clin Invest. 2014. doi: $10.1172 / \mathrm{JCl} 73455$

13. Hingorani SR, Harris WP, Beck JT, et al. Phase lb study of PEGylated recombinant human hyaluronidase and gemcitabine in patients with advanced pancreatic cancer. Clin Cancer Res. 2016. doi: 10.1158/10780432.CCR-15-2010.

14. Valentini V, Calvo F, Reni M, et al. Intra-operative radiotherapy (IORT) in pancreatic cancer: joint analysis of the ISIORT-Europe experience. Radiother Oncol. 2009. doi: 10.1016/j.radonc.2008.07.020.

15. Yu X, Di Y, Xie C, et al. An in vitro and in vivo study of gemcitabine-loaded albumin nanoparticles in a pancreatic cancer cell line. Int J Nanomed. 2015;10:6825-6834.

16. Tanaka T, Yamamoto K, Kichikawa K. Pharmacokinetic evaluation of pancreatic arterial infusion chemotherapy after unification of the blood supply in an animal model. J Vasc Interv Radiol 2010;21:116-121.

17. Reed RA, Teitelbaum GP, Daniels JR, Pentecost MJ, Katz MD. Prevalence of infection following hepatic chemoembolization with cross-linked collagen with administration of prophylactic antibiotics I. J Vasc Interv Radiol. 1994;5:367-371.

18. Roychowdhury DF, Cassidy CA, Peterson $P$, et al. A report on serious pulmonary toxicity associated with gemcitabine-based therapy. Invest New Drugs. 2002;20:311-315.
19. Rasheed ZA, Matsui W MA. Pathology of pancreatic stroma in PDAC. In: Pancreatic Cancer and Tumor Microenvironment, 1st ed. Grippo PJ, Munsh HG (eds.). Trivandrum, India: Transworld Research Network; 2012.

20. Feig C, Gopinathan A, Neesse A, et al. The pancreas cancer microenvironment. Clin Cancer Res. 2012;18:4266-4276.

21. Tozzi A, Comito T, Alongi $F$, et al. SBRT in unresectable advanced pancreatic cancer: preliminary results of a mono-institutional experience. Radiat Oncol. 2013:1-8.

22. Miyanishi $\mathrm{K}$, Ishiwatari $\mathrm{H}$, Hayashi $\mathrm{T}$, et al. A phase i trial of arterial infusion chemotherapy with gemcitabine and 5-fluorouracil for unresectable advanced pancreatic cancer after vascular supply distribution via superselective embolization. Jpn J Clin Oncol. 2008. doi: 10.1093/jjco/hyn015.

23. Tanaka $T$, Sho $M$, Nishiofuku $H$, et al. Unresectable pancreatic cancer: arterial embolization to achieve a single blood supply for intraarterial infusion of 5-fluorouracil and full-dose IV gemcitabine. Am J Roentgenol. 2012;198:1445-1452.

24. Liu F, Tang Y, Sun J, et al. Regional intra-arterial vs. systemic chemotherapy for advanced pancreatic cancer: a systematic review and metaanalysis of randomized controlled trials. PLoS One. 2012. doi: 10.1371/ journal.pone.0040847.

25. Oderich GS, Tallarita T, Gloviczki P, et al. Mesenteric artery complications during angioplasty and stent placement for atherosclerotic chronic mesenteric ischemia. J Vasc Surg. 2012;55:1063-1071.

26. Alvarez-Gallego R. Pathological response to neoadjuvant gemcetabine and nabpacxitaxol in pancreatic adenocarcinoma to imrove survival. J Clin Oncol. 2016;34:abstract 4109.

Cite this article as: Rosemurgy AS, Ross SB, Vitulli PL, Malek R, Li J, Agah R (2017) Safety study of targeted and localized intra-arterial delivery of gemcitabine in patients with locally advanced pancreatic adenocarcinoma, Journal of Pancreatic Cancer 3:1, 58-65,

DOI: 10.1089/pancan.2017.0011.

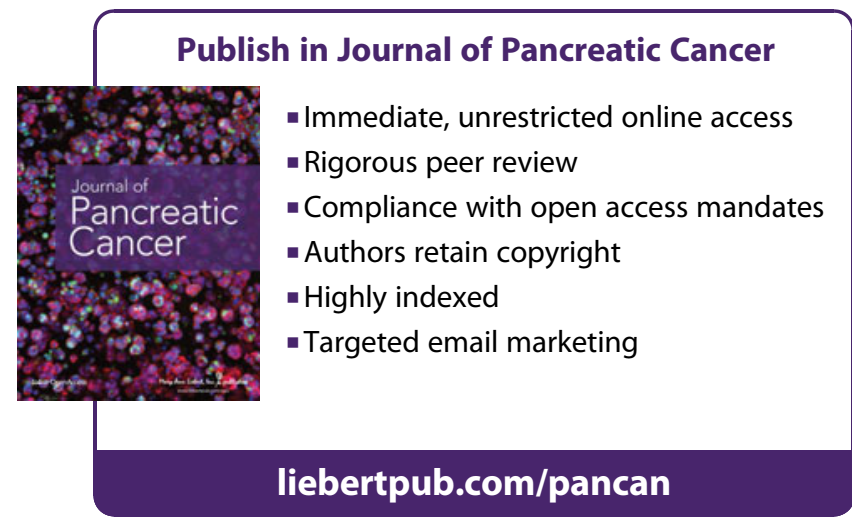

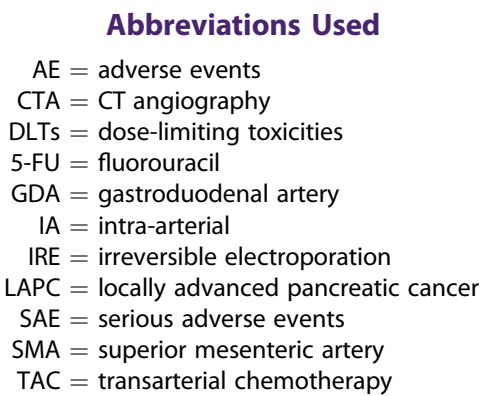

liebertpub.com/pancan 